\title{
Objective Analysis of HDTV H.264 x MPEG-2 with and Without Packet Loss
}

\author{
Eduardo Santos Bueno, Cristiano Akamine, Renato de Mendonça Maroja, and Gustavo de Melo \\ Valeira
}

\begin{abstract}
This article aims to compare video quality between H.264 and MPEG-2 compression methods through an objective analysis. The method used to assess the video in the test is through comparison with the reference video. The tests were conducted using Picture Quality Analysis (PQA) equipment. The PQA has the following methods for an objective evaluation: Picture Quality Rating (PQR) and Differential Mean Opinion Score (DMOS). The PQR and DMOS methods are objective evaluations approaching subjective evaluations.

By only analyzing the video compression imperfections, i.e., no packet loss, it can be seen that $\mathbf{H . 2 6 4}$ presents video quality equivalent to MPEG-2 with a transmission rate about two times smaller. However, the study completed for this article relates to video quality with packet loss in the transport layer of the ISDB$T_{B}$ transmission system, this shows that H.264 suffers a bigger loss in video quality than MPEG-2 with the same value in relation to signal-to-noise ratio (SNR) in the transmission channel, into the ISDB- $T_{B}$ receiver. To generate error packets in the transmission, white noise was added between the transmitted and received signal. The test results show that $\mathrm{H.264}$ provides superior video quality when compared to MPEG-2, with no packet loss, but with packet loss, MPEG-2 provides better video quality than H.264.
\end{abstract}

Index Terms-Differential Mean Opinion Score (DMOS), H.264, MPEG-2, Packet loss, Picture Quality Analysis (PQA), Picture Quality Rating (PQR).

\section{INTRODUCTION}

$\mathrm{T}$ ERRESTRIAL digital television was first broadcast in Brazil in December 2007 in São Paulo; it is based on the Japanese digital television system Integrated Services Digital Broadcasting - Terrestrial (ISDB-T). The main differences between the systems adopted in both countries are the audio/video encoding, middleware, and channel allocation [1] [2] [3].

The video compression system used by the Brazilian system is the Moving Picture Expert Group (MPEG-4 part 10) known as H.264 and MPEG-2 is another encoding method, which is used in other digital television systems like the Advanced

Eduardo Santos Bueno is a researcher at Mackenzie Presbyterian University, São Paulo, Brazil (e-mail: duardo.bueno@mackenzie.br).

Cristiano Akamine is a professor at Mackenzie Presbyterian University, São Paulo, Brazil (e-mail: cristiano.akamine@mackenzie.br).

Renato de Mendonça Maroja is a researcher at Mackenzie Presbyterian University, São Paulo, Brazil (e-mail: rmmaroja@gmail.com)

Gustavo de Melo Valeira is a researcher at Mackenzie Presbyterian University, São Paulo, Brazil (e-mail: gustavo.valeira@mackenzie.br).
Television System Committee (ATSC), Digital Video Broadcasting (DVB), and ISDB-T.

Recommendation H.264 is a document published by the International Telecommunication Union (ITU) and International Organization for Standardization / International Electrotechnical Commission (ISO/IEC) [4] [5].

The H.264 standard was published in 2003 and is based on the concepts of previous standards, such as MPEG-2 and MPEG-4 part 2, and offers better efficiency in video compression, i.e., better image quality and more flexibility in compression, transmission, and video storage [6]. H.264 can be used in many applications, including video conferencing, television transmission, and data storage. Therefore, it is obtained using a variety of video compression algorithms that compact an image sequence forming a digital video that uses a lower bit transfer rate than the original video.

However, with a constant increase in the information to be transmitted, there is also a need for greater video compression, which can cause minor degradation in the picture, but it is possible to assess this loss in image quality through a subjective or objective evaluation.

The criteria used in a subjective evaluation of image quality must comply with the standards established by ITU-R BT.50011 [7].

In video compression, the criteria used in an objective evaluation of picture quality must be in accordance with the standards established by the ITU and ISO/IEC, which are both independent organizations for the global standardization of telecommunications.

The objective of this study is to compare the objective video quality of video compression methods H.264 and MPEG-2. The objective comparison was carried out using the Picture Quality Analysis (PQA) equipment, and the metrics used were Picture Quality Rating (PQR) and Differential Mean Opinion Score (DMOS).

This required using video encoders for both H.264 and MPEG-2 at different compression rates. From the results, it was possible to assess the performance of each compression system by the video rate, and so compare H.264 in relation to MPEG-2.

Several articles in the literature say the H.264 video compression method is more efficient than MPEG-2 [4] [6] [8]. Therefore, this study quantifies these differences. Knowing these differences, it is possible to determine the quality gain versus bit rate. 


\section{OBJective Measures}

In a video system, there are many video processing devices that can affect image quality, including the stages of encoding and decoding, which can cause some image degradation.

It is considered that the best assessment of video quality is done by human evaluators since the important thing is to meet the viewer's needs. However, assessing video quality by a subjective evaluation requires a large amount of time and has a high cost. Furthermore, the subjective analysis can only detect visible degradation, and result variations occur due to the evaluators. Thus, what is needed is the development of objective evaluation methods for video quality based on subjective image analysis, and the methods used in this paper are based on this.

The objective analysis of the image quality can be performed with the PQA equipment that can perform the PQR and DMOS measurements.

The PQR and DMOS methods analyze the perceptual contrast difference between a reference video and a test video. The PQR measurement is an assessment of image quality, which corresponds to the perceptual sensitivity measurement that only determines noticeable image differences, known as Just Noticeable Differences (JND) [9] [10].

The JND is based on human perception, which assesses the human reaction to variations in image quality so that it is possible to assess the quality of an image in relation to another [8]. For example, to measure JND, two images or videos are compared, i.e., a reference video and a test video, where this video is derived from the reference video, which in turn has some defects. The lower the JND value, the lower the differences between the test video and the reference video are. 1 JND corresponds to $1 \mathrm{PQR}$.

A difference of $1 \mathrm{JND}$ is approximately $0.1 \%$ of the contrast difference perception between the reference video and the test video [10] [11].* However, with this perceptive contrast difference, viewers do not easily distinguish which is the reference video and which is the test video, therefore, they are practically equal.

However, the perceptual contrast difference between a reference video and a test video becomes clearer with values above $2 \mathrm{JND}$. If the reference video and the test differ by 3 JND or more, viewers will always notice the video differences, in $100 \%$ of cases [10] [11].

It can be concluded that the smaller the PQR, the better the quality of the test video with respect to the reference video will be.

The DMOS measurement corresponds to the subjective evaluations of picture quality, which are completed by evaluators, using the ITU procedure ITU-R BT.500. This is possible because the PQA generates a map of the perceptual contrast differences between the reference video content and the test, which contains information about the differences that viewers will notice between the reference video and the test video [10] [11].

However, unlike subjective image analysis, in which evaluators are used, PQA produces a DMOS result for the sequence of each picture frame. Thus, the DMOS analyzes the contents of the entire video.
The grade is given, based on a five-point scale to assess both the reference video and the test video as in Fig. 1 [10] [11].

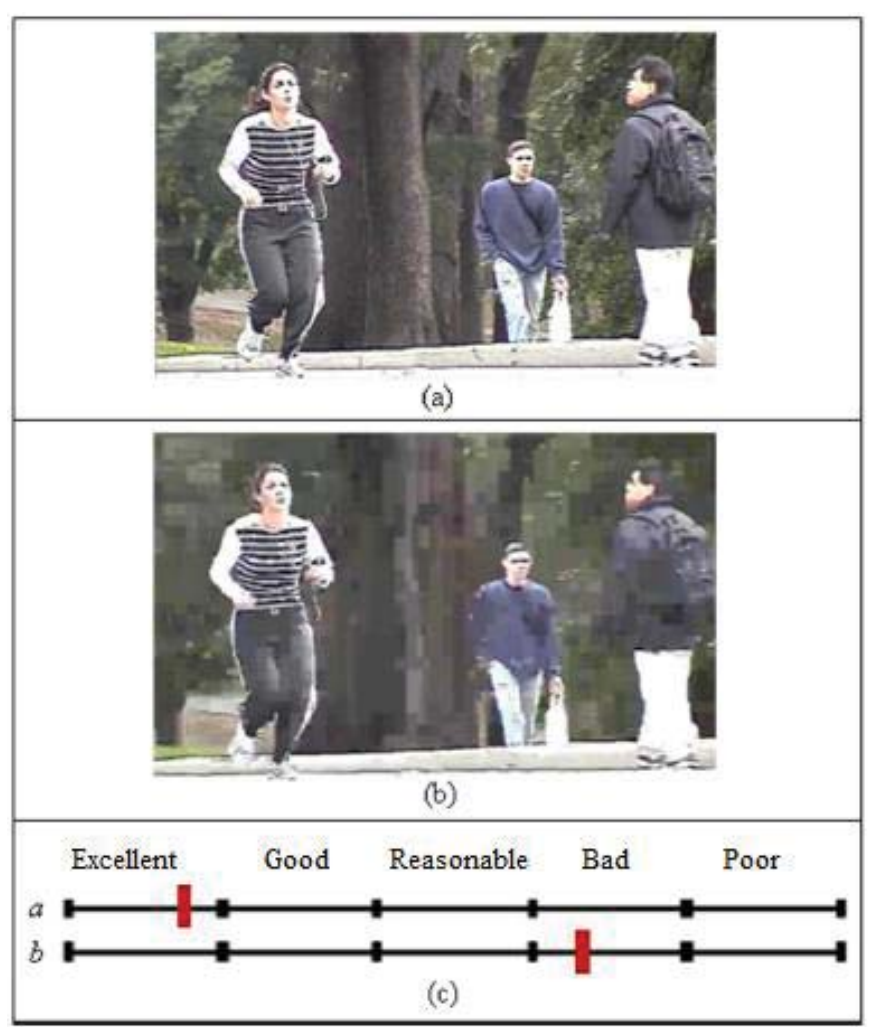

Fig. 1. Demonstration DMOS assessment (a) reference video, (b) test video and (c) note scale. Source - Tektronix - PQA500.

In Fig. 1 (c) the $a$ scale is for reference video and the $b$ scale is for the test video. The scale in Fig. 1(c) refers to the video quality of Fig. 1(a) and Fig. 1(b). The grade is displayed with a mark added to the scale. From the results, the Mean Opinion Score (MOS) is calculated, i.e., the average result given by the evaluators. The values obtained using the scale are converted into a numerical value, which is the score that the PQA analyzed. To obtain the DMOS, it is necessary to subtract the reference video MOS from the test video's MOS [10] [11].

Values between 0-20 are classified as "excellent" and the test video is good in relation to the reference video [10] [11].

Values between 21-40 mean that the test video is "good" and the video quality is fairly good compared to the reference video [10] [11].

Values between 41-60 indicate that the test video is "reasonable" and the video quality is only acceptable in relation to the test video, but the quality is not good, so the viewer feels uncomfortable when watching the video [10] [11].

Values between 61 to 80 are categorized as "bad" and the test video quality is simply bad in relation to the reference video. For this reason, the image defects are clearly perceived [10] [11].

Values over 81 indicate that the test video has several defects and is classified as "poor" [10] [11]. 


\section{Test Model OBJective}

The test was designed to objectively analyze video quality for the following variables: encoding standard, bit rate without packet loss, and bit rate with packet loss. For each of the video encodings H.264 and MPEG-2, the bit rates selected were 3 $\mathrm{Mb} / \mathrm{s}$ to $15 \mathrm{Mb} / \mathrm{s}$ with intervals of $1 \mathrm{Mb} / \mathrm{s}$. With packet loss, the bit rate chosen was $13 \mathrm{Mb} / \mathrm{s}$ with a variation in the signalto-noise ratio between $17.9 \mathrm{~dB}$ and $17.2 \mathrm{~dB}$ with an interval of $0.1 \mathrm{~dB}$. Tests were performed on three different videos without being compressed, as in Table I. The HDTV video sources are available from Tektronix.

TABLE I

DESCRIPTION OF VIDEOS

\begin{tabular}{ccccc}
\hline \hline Name & Size & Time & Frames & Description \\
\hline $\begin{array}{c}\text { Eighth } \\
\text { Ave }\end{array}$ & $5.11 \mathrm{~GB}$ & $44.18 \mathrm{~s}$ & 1325 & $\begin{array}{c}\text { A video that has } \\
\text { many people } \\
\text { walking on a } \\
\text { boulevard. However, } \\
\text { the camera is stable. }\end{array}$ \\
$\begin{array}{c}\text { Stripy } \\
\text { jogger }\end{array}$ & $1.87 \mathrm{~GB}$ & $16.18 \mathrm{~s}$ & 486 & $\begin{array}{l}\text { The camera spends } \\
\text { all his time focused } \\
\text { on a woman running } \\
\text { in a park, therefore, } \\
\text { has the movement of } \\
\text { people and the } \\
\text { camera. }\end{array}$ \\
\hline $\begin{array}{c}\text { Times } \\
\text { Square }\end{array}$ & $2.94 \mathrm{~GB}$ & $25.43 \mathrm{~s}$ & 763 & $\begin{array}{c}\text { It has no movement } \\
\text { in the video content } \\
\text { only camera circular } \\
\text { motion when } \\
\text { shooting buildings. }\end{array}$ \\
\hline \hline
\end{tabular}

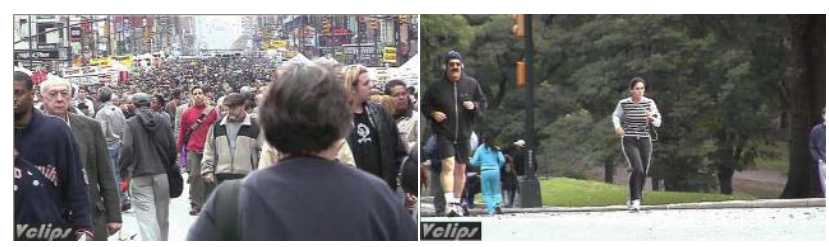

Eighth Ave Stripy jogger

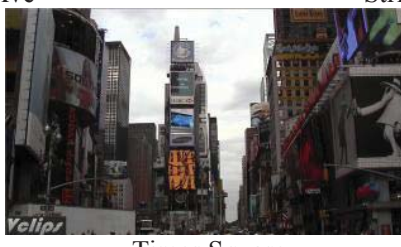

Times Square

Fig. 2. One frame from each of the 3 video sources used. Source - Tektronix - PQA500.

The tests were conducted using the full-reference measuring method. The full-reference measurement compares the reference video sequence with the test video sequence. The reference video is the original video, and the test video is the video after the encoding of the reference video, and the comparisons are made frame by frame.

The MPEG-2 and H.264 encodings are performed with the use of encoders in real time, i.e., the same type used by television broadcasters. For the Group of Picture (GOP) configuration, the values used were values recommended by the encoders of HDTV encoding. For MPEG-2, a distance was adopted for frame I (with 5 frames), and another distance for frame P (with 3 frames). For H.264, a limited distance was adopted for frame I (between 1 and 255 frames), and for frame $\mathrm{P}$ (between 1 and 3 frames).

The MPEG-2 and H.264 encodings were performed with the standard configuration profile/High for video encoding 1080i 30fps (i.e. 1920 pixels by 1080 lines interspersed, 30 frames per second).

The first test was to verify the video quality of the MPEG-2 and H.264 encoders without packet loss. The setup for this test is shown in Fig. 3.

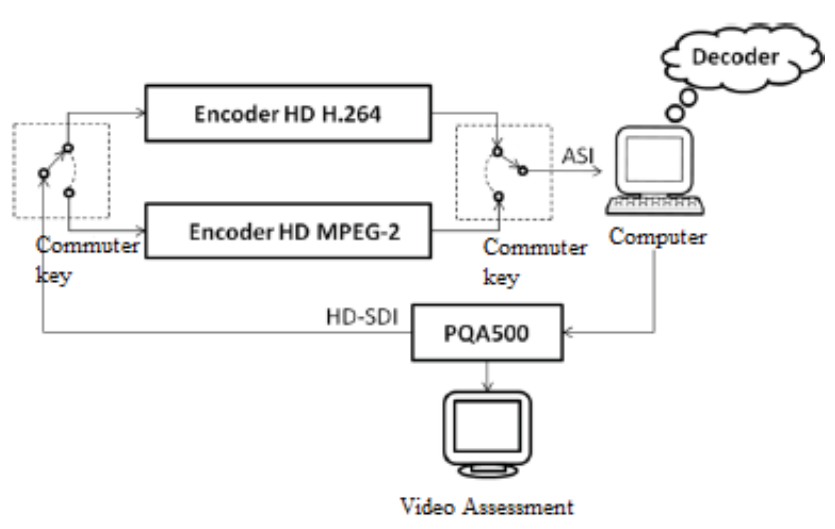

Fig. 3. Block diagram of the first test configuration

As shown in Fig. 3, PQA generates the reference video in a HD signal format by the High Definition - Serial Digital Interface (HD-SDI) which is then encoded by one of the encoders. The encoding is performed on both H.264 and MPEG-2 separately. The computer has a card that receives the Transport Stream (TS) via the serial interface known as Asynchronous Serial Interface (ASI) and writes the TS. This operation is performed at various compression rates and after recording the compressed videos, they are decoded for comparison with the original video, in PQA.

In the first test, the three encoded video sequences shown in Fig. 2 were encoded, which only allowed the comparison of encoding quality, with different bit rates. The decoding was performed in the software.

The second test was to determine how much video quality degradation occurred using MPEG-2 and H.264 encoders when there is packet loss in the transport layer of the ISDB- $T_{B}$ transmission system. To generate error packets in the transmission, white noise was added to the modulated signal applied to the receiver. The use of white noise is effective because it has a constant spectrum along the frequency. The setup for this test is shown in Fig. 4. 


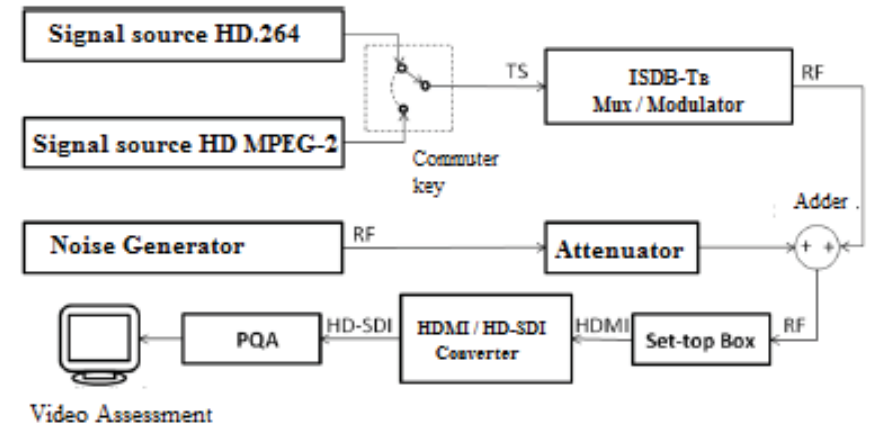

Fig. 4. Block diagram of the second test configuration

In Fig. 4, the signal source generates a HD video in both the H.264 standard and in MPEG-2. In both compression methods, a bit rate of $13 \mathrm{Mb} / \mathrm{s}$ has been used. The encoded video signal is multiplexed and modulated in the ISDB- $\mathrm{T}_{\mathrm{B}}$ transmission system, to be received by the set-top box. The transmission configuration used is shown in Table II. However, to generate error packets in the transmission channel, white noise is added to the signal. PQA receives the signal decoded from the Set-Top Box, which was necessary to convert High-Definition Multimedia Interface (HDMI) to HDSDI. This operation is performed with various signal-to-noise ratio values (SNR). After PQA receives the video, the comparison of the encoded video is performed at a rate of 13 $\mathrm{Mb} / \mathrm{s}$, but with some packet loss.

TABLE II

ISDB- $\mathrm{T}_{\mathrm{B}}$ TRANSMISSION CONFIGURATION

\begin{tabular}{ll}
\hline \hline Mode & 3 \\
\hline Guard Interval & $1 / 16$ \\
\hline Layer & 1 \\
\hline Hierarchical layer digital modulation & $64 \mathrm{QAM}$ \\
\hline Temporal interleaver & $0.2 \mathrm{~s}$ \\
\hline Convolutional encoder rate & $3 / 4$ \\
\hline \hline
\end{tabular}

The completion of the objective tests was completed with PQA using the PQR and DMOS methods.

\section{RESUlts ANALYSis}

The video quality analysis was performed considering an ideal communication channel and a channel with varying SNR. The variation of SNR causes bit errors within the ISDB$\mathrm{T}_{\mathrm{B}}$ transport layer of the digital channel. The results obtained are presented in the next section:

\section{A. Assessment without packet loss}

The tests were performed with three video sequences and present the average results for both the PQR and DMOS methods.

Fig. 5 shows the graph for the PQR method, comparing the H.264 (blue line) and MPEG-2 (red line) encodings with a bit rate variation.

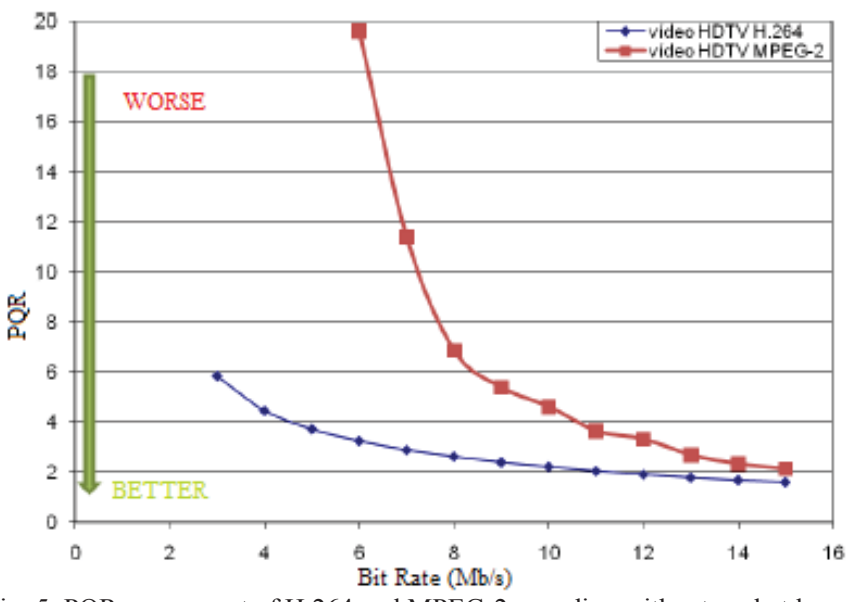

Fig. 5. PQR assessment of H.264 and MPEG-2 encoding without packet loss

As shown in Fig. 5, the H.264 encoding method is of a better quality than the MPEG-2 encoding at any compression ratio for HD video. Therefore, it is possible to transmit H.264 video at $7 \mathrm{Mb} / \mathrm{s}$ with the same quality of MPEG-2 video at 13 $\mathrm{Mb} / \mathrm{s}$.

It can be concluded that the HD video with MPEG-2 encoding is of a good quality with a low bit rate. However, the HD video with H.264 encoding is of a good quality in relation to the original video with a rate higher than $11 \mathrm{Mb} / \mathrm{s}$ with a score between 1 and $2 \mathrm{PQR}$, where the difference between the test video and the original video is barely noticeable when compared.

Fig. 6 shows the DMOS method graph, comparing the H.264 (blue line) and MPEG-2 (red line) encodings with a bit rate variation.

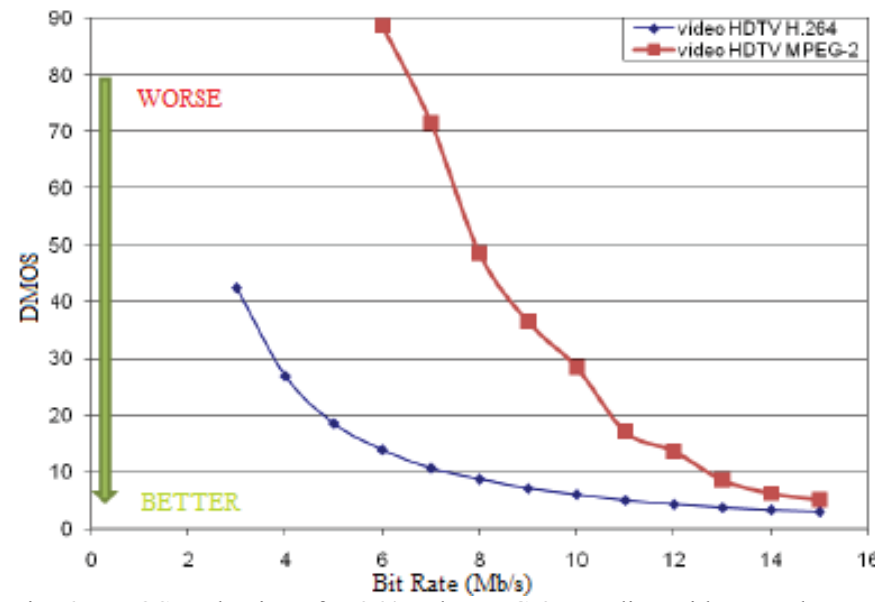

Fig. 6. DMOS evaluation of H.264 and MPEG-2 encoding without packet loss

As shown in Fig. 6, the H.264 encoding method is of a better quality than the MPEG-2 encoding at any compression ratio for HD video. Therefore, it is possible to transmit H.264 video at $7 \mathrm{Mb} / \mathrm{s}$ with the same quality of MPEG-2 video at approximately $13 \mathrm{Mb} / \mathrm{s}$.

It can be seen that the HD video with MPEG-2 encoding is not of good quality with a low bit rate. However, the HD video with H.264 encoding is of a good quality in relation to the original video with a rate higher than $8 \mathrm{Mb} / \mathrm{s}$ and is of an 
excellent quality, while the MPEG-2 acquires this degree of quality from $13 \mathrm{Mb} / \mathrm{s}$.

\section{B. Assessment with packet loss}

The following tests were performed with the three videos shown in Fig. 2. Despite using the same video sequences, for these tests they were recorded with 1800 frames, lasting 60 seconds, with a rate of $13 \mathrm{Mb} / \mathrm{s}$, to achieve this the same video was repeated a few times. In addition, various tests were completed with the same videos and the results shown are the average for each video for both the PQR and DMOS methods.

Figs. 7 and 8 show graphs for the PQR and DMOS assessment results, respectively, comparing H.264 (blue line) and MPEG-2 (red line) encodings with a change in signal-tonoise ratio.

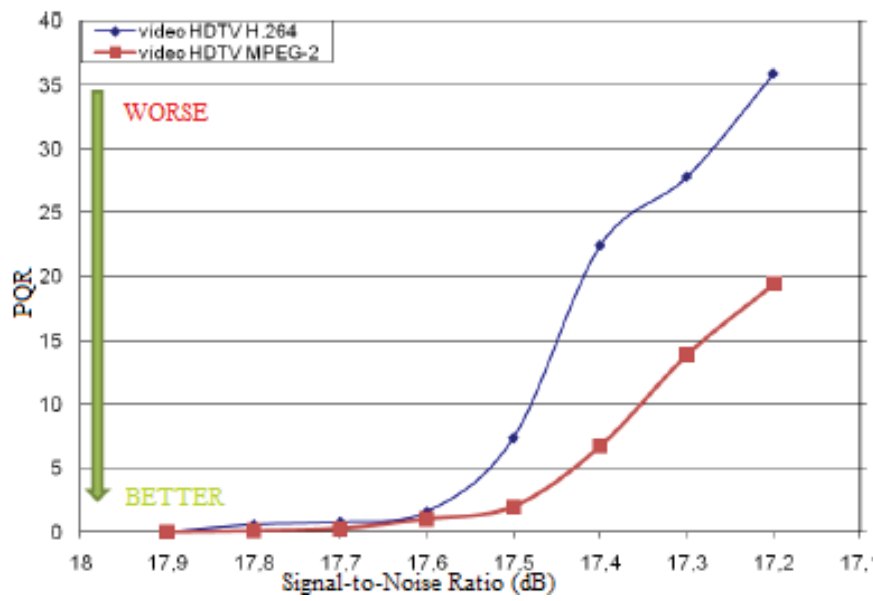

Fig. 7. PQR Evaluation of H.264 and MPEG-2 encoding with packet loss

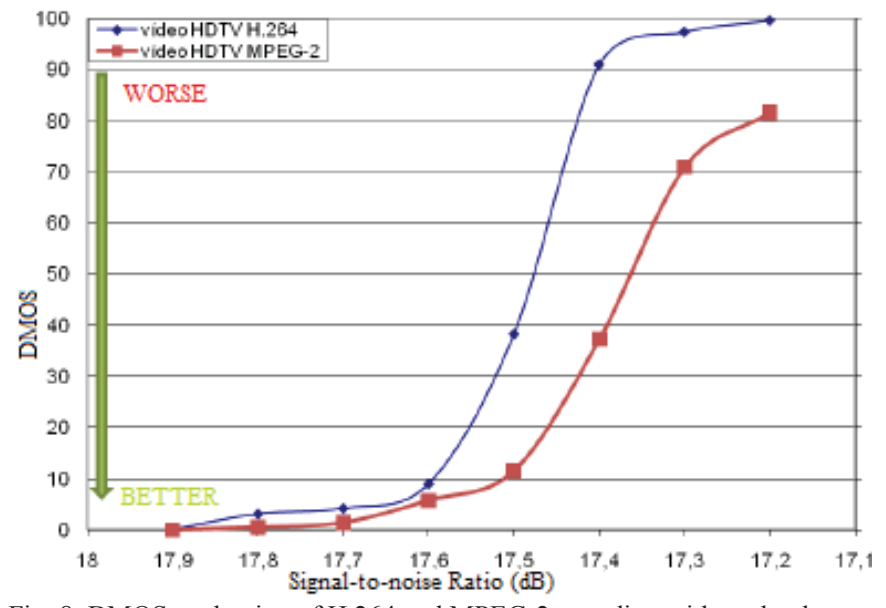

Fig. 8. DMOS evaluation of H.264 and MPEG-2 encoding with packet loss

These results show that H.264 is more sensitive to packet loss than MPEG-2. It is observed that when the SNR value is between $17.9 \mathrm{~dB}$ and $17.6 \mathrm{~dB}$ the encoders are similarly affected. However, H.264 is shown to be more sensitive to packet loss than MPEG-2, from an SNR value of $17.6 \mathrm{~dB}$.

This occurs because the H.264 decoder is slower to recover from packet loss. Moreover, the degradation shown by the
MPEG-2 decoder in response to packet loss had a minor impact.

\section{CONCLUSION}

The PQR and DMOS objective evaluations ensure accurate control of video quality. The DMOS assessment helps determine the differences in the test video in relation to the reference video based on subjective quality ratings. Therefore, the PQR measurement helps determine how viewers perceive the differences in the test video in relation to the reference video, an efficient high-quality video, when the differences are close to the visibility threshold.

The test results are consistent, where the H.264 encoding provides a similar quality to the MPEG-2 encoding at approximately half the bit rate. However, H.264 decreases its advantage at a high bit rate, namely above $15 \mathrm{Mb} / \mathrm{s}$, here there is little difference between MPEG-2 and H.264.

However, packet loss in test results also demonstrates that the H.264 encoding quality drops sharply while the quality of MPEG-2 encoding in response to packet loss had a minor impact. This sensitivity seems to be partly caused by the GOP structure, where the H.264 decoder takes a longer time to recover. This can be seen when viewing the number of wrong frames that the video encoded in H.264 generates when packets are lost, whereas the video encoded in MPEG-2 was shown to be better.

\section{ACKNOWLEDGMENT}

The authors would like to thank their friends in the Laboratorio de Televisão Digital at Universidade Presbiteriana Mackenzie.

\section{REFERENCES}

[1] Televisão digital terrestre - Codificação de vídeo, áudio e multiplexação Parte 1: Codificação de vídeo, ABNT NBR 15602-1:2007, Abr. 2007.

[2] Televisão digital terrestre - Codificação de vídeo, áudio e multiplexação Parte 2: Codificação de áudio, ABNT NBR 15602-2:2007, Abr. 2007.

[3] Televisão digital terrestre - Sistema de transmissão, ABNT NBR 15601:2007, Abr.2007.

[4] B. Farmer, "Video Compression using ITU-T Recommendation H.264ll." M.S Dissertation, Faculty of Engineering \& Surveying. University of Southern Queensland, Queensland, 2005.

[5] Information technology, Coding of audio-visual objects - Part 10: Advanced Video Coding. n.2, ISO/IEC 14496-10, 2004.

[6] I. E. G. Richardson, H.264 and MPEG-4 Video Compression. England: Wisley, 2003, p. 281.

[7] Methodology for the subjective evaluation of the quality of television pictures, ITU-R BT.500-11, 2002.

[8] R. Arthur, "Avaliação Objetiva de Codecs de Vídeo," M.S. Dissertation, Faculdade de Engenharia Elétrica e de Computação. Universidade Estadual de Campinas. São Paulo, 2002.

[9] Measuring and Interpreting Picture Quality in MPEG Compressed Video Content. Tektronix. S.L. 2001. Available at:

$<$ http://www.tek.com/Measurement/App_Notes/25_14675/eng/25W_14675_0 .pdf $>$

[10] Perceptual-Based Objective Picture Quality Measurements. Tektronix. S.L. 2008.

[11] Understanding PQR, DMOS, and PSNR Measurements. Tektronix. S.L. 2008.

Cite this article:

Santos Bueno, E., Akamine, C. , de Mendonça Maroja, R., and de Melo Valeira, G.; 2015. Objective Analysis of HDTV H.264 x MPEG-2 with and Without Packet Loss. ISSN Print: 2446-9246 ISSN Online: 2446-9432. doi: 10.18580/ setijbe.2015.5 Web Link: http://dx.doi.org/10.18580/setijbe.2015.5 